\title{
RADIOKINETIC EVIDENCES FOR THE BIPARTITE OR DIPLOID NUCLEUS IN CONIDIA OF STREPTOMYCES GRISEOFLAVUS
}

\author{
HIUGA SAITO and YONOSUKE IKEDA \\ Division of Microbial Genetics, Institute of Applied \\ Microbiology, University of Tokyo \\ Received for publication Oct. 10, 1957
}

In the course of work attempted for isolating auxotrophic mutants of Streptomyces griseoflavus, it was found that the frequency of mutant recovery was significantly increased by incubating conidia on a complete medium after ultraviolet treatment, that is, when $\mathrm{uV}$-irradiated conidia were preincubated on the complete medium for a week before letting the newlyformed conidia stand in a minimal medium for the filtration process, about $1 \%$ of the conidia existing in the filtrate was auxotrophic, while the frequency was approximately $0.1 \%$ when tested without preincubation. Though the mechanism of preincubation effect was unclarified, the authors referred to the notion that this strain might contain two sets of genetic units in a conidium and that the so-called segregation delay might be the most possible mechanism $^{(6)}$.

When the conidia were irradiated by X-rays instead of ultraviolet light in the expectation of high frequency of induced mutation, survival curves having a shoulder in the early period of irradiation and resembling to "two-hit" curves have resulted. In the present paper, further analysis of $\mathrm{X}$-ray inactivation will be presented as another evidence for supporting the view that the nucleus in a conidium of Str. griseoflavus has replication of genetic units. It is likely that the nucleus is bipartite or diploid, because, according to the cytological studies that are to be published elsewhere, the conidium is apparently uninuclear ${ }^{(7)}$.

\section{MATERIALS AND METHODS}

Microorganisms. A laboratory strain of Streptomyces griseoflavus, No. 142 , and the two induced mutants, No. 21 dependent of isoleucine and methionine and No. 305 dependent of cystine, were employed for this study. Details of the history and characters of these strains were reported elsewhere $^{(6)(8)}$. Besides these, three species of Streptmyces fungi, Str. griseus Waksman No. 1, Str. kitasatoensis, and Str. coelicolor $1^{+}$(available through the courtesy of Dr. G. Sermonti) were used for the comparative study.

Media. Sporulation and stock media used for Str. griseoflavus and Str. griseus were of a modified Krainsky type supplemented with yeast extract 
and peptone. For colony counting, bouillon-glucose medium was employed. The conidia of Str. coelicolor were harvested from the culture on a Czapek type medium. The composition of these media were the same as described previously ${ }^{(6)}$.

The medium used for the sporulation of Str. kitasatoensis was as follows: glucose $5 \mathrm{~g}$., starch $25 \mathrm{~g}$., soybean meal $20 \mathrm{~g}$., dry yeast $5 \mathrm{~g}$., urea $0.5 \mathrm{~g},\left(\mathrm{NH}_{4}\right)_{2} \mathrm{SO}_{4} 5 \mathrm{~g}$., $\mathrm{NaCl} 5 \mathrm{~g}$., $\mathrm{K}_{2} \mathrm{HPO}_{4} 1 \mathrm{~g}$., $\mathrm{CaCO}_{3} 3 \mathrm{~g}$., agar $1.8 \mathrm{~g}$., and water $1,000 \mathrm{ml}$. (pH 7.2 with $5 \mathrm{~N}-\mathrm{NaOH})$.

Experimental procedures. Conidia harvested from 6 to 10 day-culture at $30^{\circ}$ (in some cases older cultures were used) on an appropriate medium were suspended in sterile saline, and vibrated vigorously for $20 \mathrm{~min}$. by an electric vibrator. Large particles were removed by filtrating the suspension through a Kaburagi glass filter G 3, No. 3. By these treatments, the frequency of single conidium in the suspension exceeded $90 \%$ of the total count. The remainder consisted of aggregates of conidia and minute hyphal fragments.

The rate of viable conidia to total conidia in the suspension deviated from 10 to $90 \%$ according to the strain and the culture-period. Ten $\mathrm{ml}$. of the suspension containing about $10^{5}$ total conidia per ml. was transferred to a sterile beaker covered with a glass plate, diameter one inch, and was exposed to $\mathrm{X}$-rays at a distance of $18 \mathrm{~cm}$. from the target of a Matsuda KXC-18 type X-ray projector which was operated at $200 \mathrm{Kvp}, 20 \mathrm{~mA}$. The $\mathrm{X}$-rays were filtered through a $1.0 \mathrm{~mm}$. alminum plate and intensity at that distance was $1,000 \mathrm{r}$ per min. The beaker containing suspention was rotated gently so as the whole area could get a constant dose during irradiation. The irradiation was made for successive $12 \mathrm{~min}$. intervals for the low dosages, and $24 \mathrm{~min}$. for high dosages. After different times of exposure, $0.1 \mathrm{ml}$. of the sample was removed, diluted with sterile saline and plated in triplicate on complete agar. The samples without exposure were taken from the original solution as the control and diluted independently in two systems so that the count might not be affected by dilution error. The survival number after irradiation was determined by counting the average number of colonies appearing after 4 to 5 days at $30^{\circ}$. Such a long incubation was necessary since in some of the irradiated conidia, germination started at a later period. Only the plates on which 20 to 300 colonies developed were counted and registered, because the number of colonies appearing was proportional to the dilution rate only in this range.

\section{RESULTS}

When the conidia of wild type strain, No. 142, of Str. griseoflavus were irradiated by X-rays, the dose-survival relationship was not exponential but rather sigmoidal, i.e., the surviving curve in a logarithmic scale plotted against the X-ray dose had a clear shoulder, and the extrapolation of the 
straight portion intercepted the ordinate at 2.3, as shown in Figure 1. This fact was confirmed by three independent experiments. The first data were obtained by the use of conidia harvested from 6 day-culture on the sporulation medium (the viability of conidia was 90\%) and the second from 25

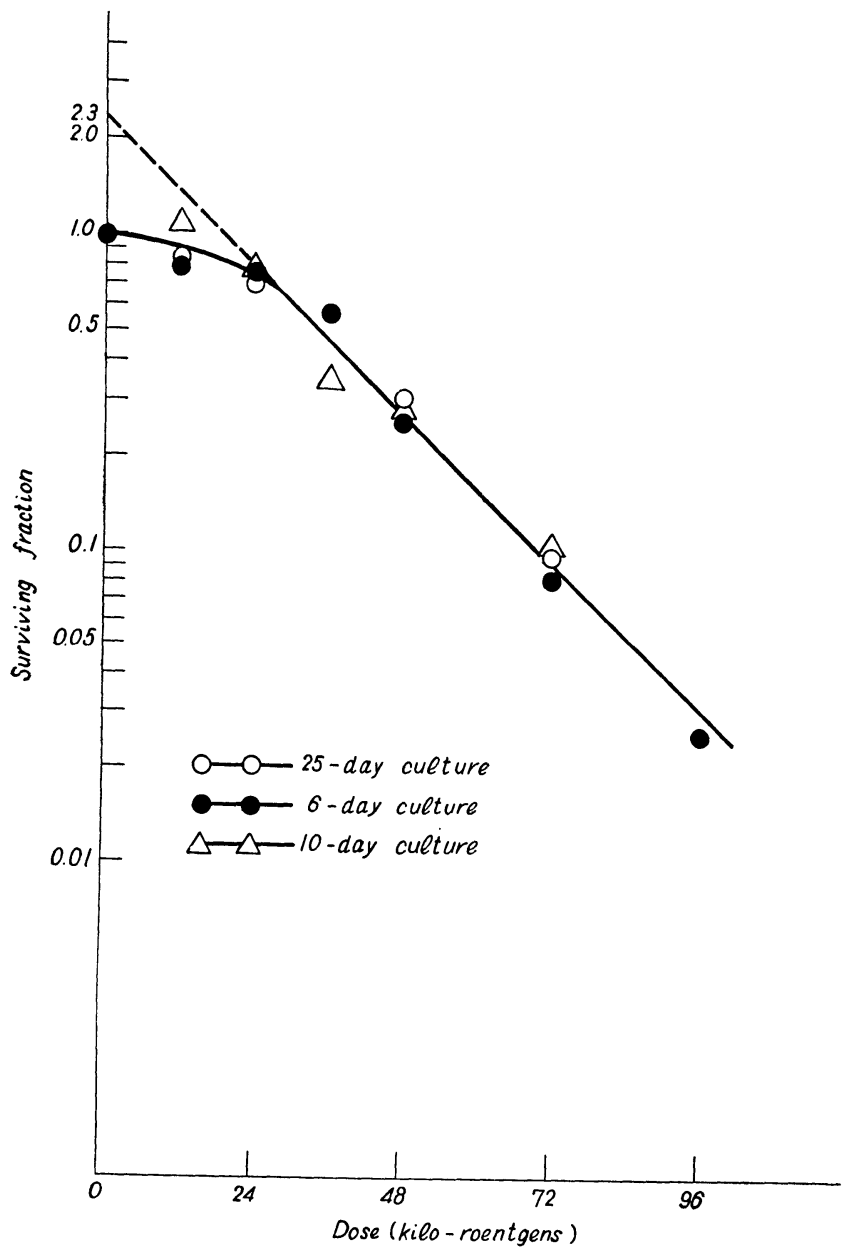

Fig. 1. Survival after X-irradiation of Str. griseoflavus.

day-culture (viability: 42\%). In the first case, the suspension with less than $2 \%$ clumping conidia were specially prepared by filtrating the conidia suspension three times. As compared with the first two, the conditions employed for third data were considerably different; the conidia were harvested from 10 day-culture, of which the viability was incidentally low (12.5\%), and suspended in saline resulting in a solution containing $10^{6}$ total conidia per $\mathrm{ml}$. This suspension was irradiated by X-rays at $5^{\circ}$, much lower than the 
temperature $\left(20-25^{\circ}\right)$ at which the former two were treated. As these three data coincided well as seen in Figure 1, it appears that the survival curve is independent of the environmental conditions and is controlled by the intrinsic character of the strain.

When two auxotrophic mutants, No. $21 i l^{*} m e_{1}$ and No. $305 c y^{*}$, both of which were induced from the strain No. 142 by $u V$-irradiation, were treated with X-rays, the survival curves shown in Figure 2 were obtained (viabilities

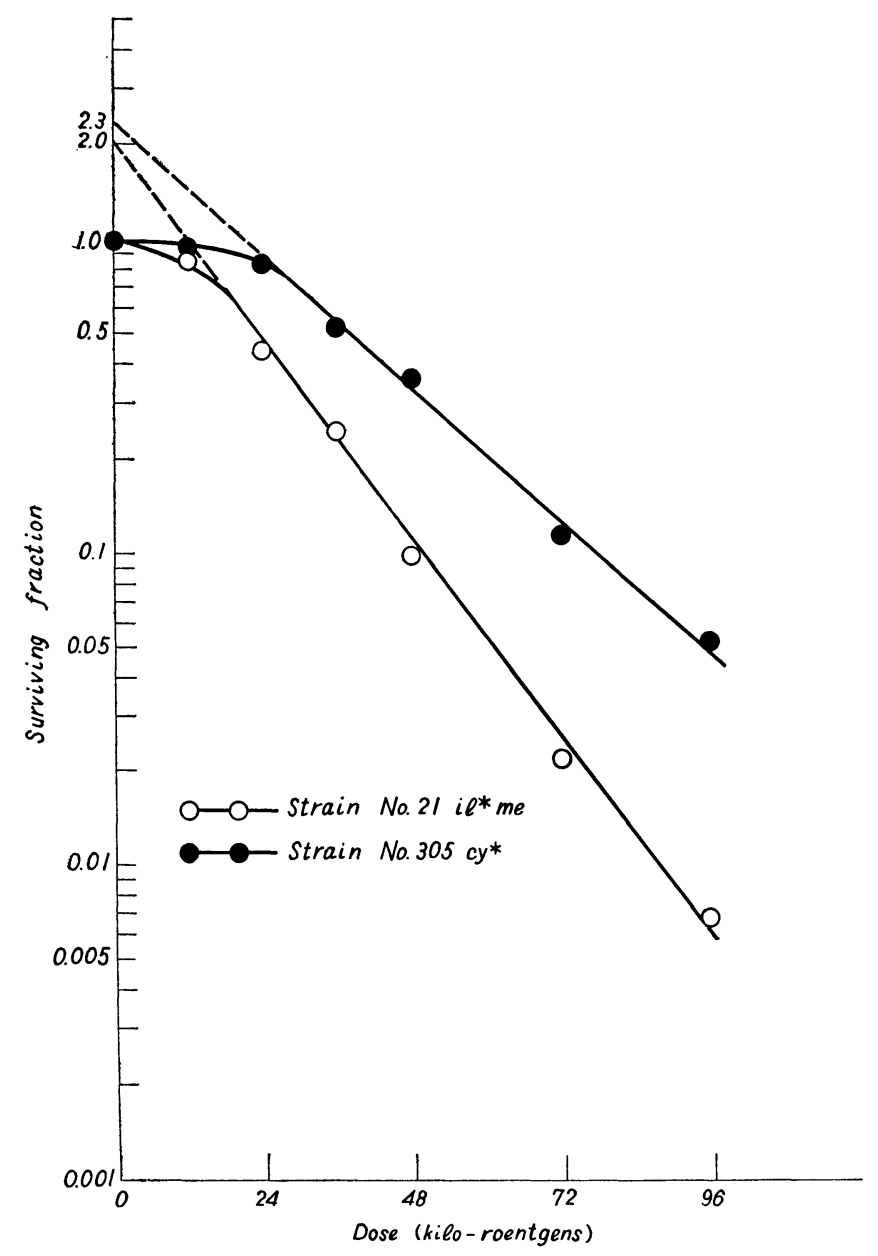

Fig. 2. Survival after X-irradiation of Auxotrophic Mutants of Str. griseoflavus.

of conidia: $79 \%$ and $50 \%$, respectively). Though the length of shoulders and the slopes of the straight portion were somewhat different from that of the strain No. 142, they were still sigmoidal and extrapolated to approximately 2 at the zero dose. Therefore, such a constant extrapolation value 
may not be caused by the reflection of experimental conditions, but rather by the genetic structure of the strain.

In order to make a comparison with Str. griseoflavus, three species of Streptomyces, Str. griseus, Str. coelicolor and St. kitasatoensis, served for the radiokinetic analysis. In the latter cases, the conidia were harvested from cultures on the Czapek's medium or on the sporulation medium specially prepared, because the strains did not form sufficient amount of conidia on the modified Krainsky's medium. By exposing the conidia of these three strains to X-rays in the same conditions as described above, three survival curves shown in Figure 3-I, II and III, were obtained. Though the curves

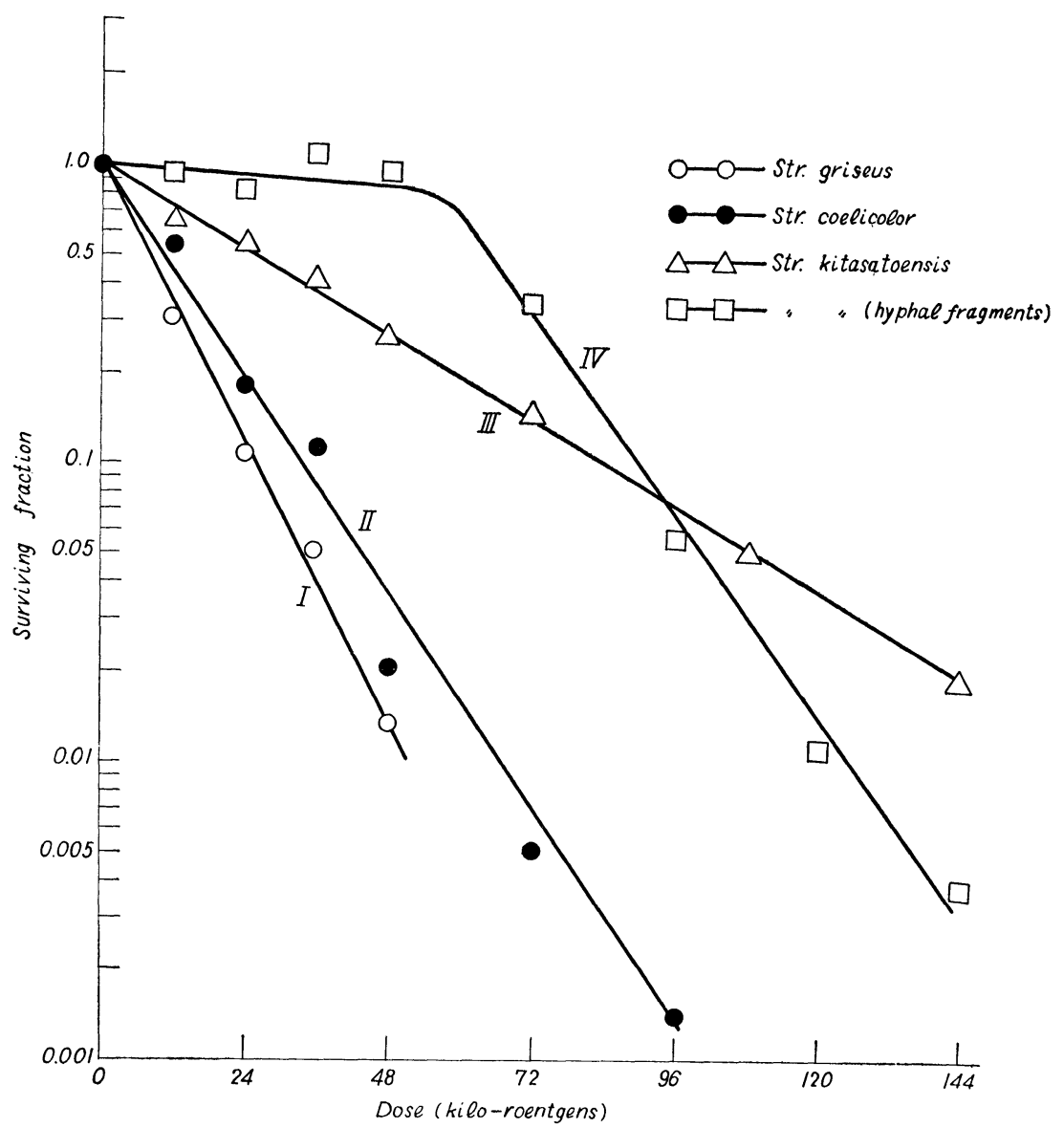

Fig. 3. Survival after X-irradiation of Str. griseus, Str. coelicolor, and Str. kitasatoensis.

have different slopes from that of Str. griseoflavus, they are apparently exponential. These results are consistent with the accepted belief that most 
species of Steptomyces as well as other microorganisms give exponential survival curves when irradiated by X-rays, and, furthermore, suggest that the sigmoidal survival curve of Str. griseoflavus may not be due to any technical error in X-ray irradiation process, but to characteristic biological behavior.

In the next experiment, X-ray inactivation test of hyphae, which was enough in length to contain several nuclei in each fragment, was attempted in the expectation of obtaining a survival curve having a shoulder if the type of curve might depend on the number of hereditary units such as nucleus in each cell. Str. kitasatoensis was used for this purpose and hyphal suspension was prepared in the following way. Many hyphal fragments were scraped off from the 10 day-culture on the modified Krainsky's medium and suspended into sterile saline; this suspension contained few conidia, since the strain hardly conidiated on this medium. Clumps of hyphae were cut into small pieces by stirring the suspension vigorously with a homogenizer. Then the hyphal fragments which were free of conidia were collected by centrifuging at a slow speed. Long fragments and remained aggregates were filtered off through a glass filter, G 3, No. 2 (not by No. 3), so that only the hyphal fragments with the length to 10 to $50 \mu$ remained in the suspension. Thus the prepared suspension was exposed to X-rays in the same way, as in the case of conidial suspension. Figure 3-IV shows one of the survival curves obtained with the hyphal fragments; it has a long shoulder as was expected. Repetition of this kind of experiment, revealed that the lengths of the shoulder were variable according to the lengths of hyphae employed. In general, however, the survival curves extrapolated to around 10 , and this value was consistent with the average number of chromatinic substances contained in the hyphae having the length of 10 to $50 \mu$.

Besides the hyphal fragments harvested from solid culture as described above, the hyphae prepared by the shaking culture resulted in a similar $\mathrm{X}$-ray inactivation curve.

\section{DISCUSSION}

During the past decades, Lea's target theory has been widely accepted in the field of radiation biology, and it has been known that the conidia of Streptomyces spp. as well as many kinds of microorganisms give exponential survival curves as typical results upon inactivation by ionizing irradiation. According to Lea ${ }^{(2)}$, the occasional finding of a sigmoidal curve in the radiokinetic study of microorganisms may be due to some disturbing factors. One possible disturbing factor may be the presence of aggregates of the cells in the suspension, but this factor might not act in the present studies, since more than $90 \%$ of conidia were exactly single in every preparation. A second disturbing factor suggestible may be the accumulation of certain toxic substances which are produced by the irradiation and may secondarily 
act upon the microorganisms. In case of small dosages, the concentrations of the poisons, if toxicity exists, would be insufficient to kill any cell, and the rate of death observed would be due to the direct action of radiation on the cells. With the increase of dosage the concentrations of the poisons become rich enough to cause appreciable mortality, and death will be accelerated by both the direct action of radiation and the secondary action caused by the poisons; thus an apparently sigmoidal curve may be resulted. In the present study, however, the conidial suspension irradiated by X-rays was considerably diluted before being plated, so that the toxic substances, if any, may not affect the germination of conidia, and besides, it is unlikely that the poisons selectively acted toxically upon Str. griseoflavus without affecting any other strains which gave exponential survival curves.

Moreover, it is not probable that the sigmoidal survival curves were produced by any accidental errors, but, on the contrary, according to the following reasons, it is considered that the curves were due to the intrinsic character of the strain. One evidence is that survival curves were highly reproducible independently of enviromental conditions, either of the culture or of the irradiation. Second, X-ray inactivation of mutant strains derived from Str. griseoflavus revealed the same type of survival curves, and were invariably extrapolated around 2 in spite of the fact that slopes of the straight portion differed so much among the wild strain and the auxotrophic mutants. As the next evidence, several other species of Streptomyces tested in the same way evidently gave exponential survival curves. This fact is consistent with the notion that most Streptomyces have a single target in one conidium, as pointed out above. As an additional study indicating the interrelation between the target theory and the biological background, it was proved that the inactivation curve of hyphal fragments was a sigmoidal one which gave the same extrapolation value as the observed number of nuclei in one fragment. For the above-mentioned reasons, the apparent "two-hit" curves obtained by irradiating the conidia of Str. griseoflavus would reflect the presence of double targets ${ }^{(1)}$, or probably double genetic units, in one conidium.

Employing a family of polyploid yeasts, Latarjet and Ephrussi, and later Lucke and Saracheck suggested that a single target sensitive to X-rays existed for each chromosome set ${ }^{(3,4)}$. According to the radiokinetic study of yeasts by Pomper et $a l^{(5)}$, some diploid strains showed exponetial curves, nevertheless they described that strains which gave "two-hit" type curves were diploid.

With reference to these preceding studies, it may be said that the conidia of Str. griseoflavus which give "two-hit" type survival curves involves double genetic units in each cell. Under this assumption, the segregation delay which was experienced in the auxotroph detection described in the previous report is readily apprehended. In this connection, it must also be commented that the cytological study of the strain reported elsewhere has 
already presented another evidence that the conidia are uninuclear and that the nucleus develops from a "dumpbell" shaped chromatinic substance which might consist of two units. For this reason, it is the conclusion of the present studies that the conidium of Str. griseoflavus may comprise a bipartite or diploid nucleus in each cell.

\section{SUMMARY}

In contrast to several species of Streptomyces which revealed exponential survival curves by X-ray inactivation tests, Str. griseoflavus and auxotrophic mutants of the strain gave apparently "two-hit" curves. On the ground of the interrelation between radiokinetic and biological findings, a proposal that the conidium of Str. griseoflavus comprises a bipartite or diploid nucleus in each cell was presented.

\section{ACKNOWLEDGEMENTS}

The authors wish to acknowledge their gratitude to Mr. S. Mashima for the technical assistance in part of this study.

\section{REFERENCES}

(1) Atwood, K. C., and A. Norman: Proc. Nat. Acad. Sci. U. S. 35, 695 (1949).

(2) Lea, D. E.: Actions of radiations on living cells, 2nd Ed. Cambridge University press, Cambridge, p. 319 (1955).

(3) Latarjet, R., and B. Ephrussi: Compt. rend. 229, 306 (1949).

(4) Lucke, W. H., and A. Sarachek: Nature 171, 1014 (1953).

(5) Pomper, S., K. M. Daniels and D. W. McKee: Genetics 39, 343 (1954).

(6) Saito, H., and Y. Ikeda: J. Gen. Appl. Microbiol. 3, 240 (1957).

(7) Saito, H., and Y. Ikeda: in preparation.

(8) Saito, H., and Y. Ikeda: J. Agr. Chem. Soc. Japan 29, 869 (1955). 